\title{
KINETICS AGGREGATION OF MAGNETIC SUSPENSIONS
}

\author{
G.Bossis $^{1}$, L.Iskakova ${ }^{2}$, V.Kostenko ${ }^{2}$ and A.Zubarev ${ }^{2}$ \\ ${ }^{1}$ Laboratoire de Physique de la Matière Condensée, CNRS UMR 6622, Université de Nice- \\ Sophia Antipolis, Parc Valrose, 06108 Nice Cedex 2, France \\ ${ }^{2}$ Ural State University, pr.Lenina 51,Ekaterinburg, Russia
}

\begin{abstract}
We present results of theoretical and computer study of the kinetics of chain-like aggregate formation in suspensions of non-Brownian magnetizable particles. An analytical model for calculation of the time-dependent function of distribution over chain size is suggested. This model describes the evolution of the chain structure due to the chain-chain aggregation. In order to verify this model we have compared it with the results of computer simulations of twodimensional model of this suspension. Results of computer simulations and of the analytical model are in reasonable agreement up to $5 \%$ of the surface concentration of the particles.
\end{abstract}

Key words: Magnetic suspension; chaining; kinetics.

\section{Introduction}

Magnetorheological suspensions (MRS) are suspensions of micron-sized magnetizable particles in a carrier liquid. Right after the first synthesis in the 40's, they attract a considerable interest of investigators and engineers due to rich set of unique physical properties valuable for many modern high technologies. In part, under the action of applied magnetic field, viscosity and other rheological properties of MRS can be changed up to several orders of magnitude. The ability to control rheological properties and behavior of MRS find active application in the machine building, instrument engineering, orthopedics and many other technologies. Overview of works on the physics of MRS and their practical applications can be found in ref. [1].

The strong dependence of the rheological and other properties of MRS on the applied field can be explained by the aggregation of the particles in linear chains, dense column-like or other heterogeneous structures aligned along the field. When these structures span the chamber with MRS and form "bridges" between the chamber walls, the rheological state of suspension changes from a flowing fluid to a quasy-elastic material. Elastic moduli of the material strongly depend on the applied field. Simultaneously with the "fluid - elastic material" transition, the suspension electro-conductivity changes up to several orders of magnitude. Both of these effects are used in many modern technologies.

Obviously, the practical applications of MRS are determined by the rate of change of their physical properties after application of the field. Thus, study of kinetics of the internal transformations in magnetic suspensions is quite important both from the scientific and practical points of view.

To our knowledge, the first model of the kinetics of evolution of the chain ensembles in the systems of magnetizable particles has been developed by M.Doi et al [2]. In this theory all chains have the same size at any given instant. Obviously, it is a very crude approximation. 
Several numerical simulations have focused on the exponent of the growth of the chain average size $S$ with time: $S(t) \propto t^{z}$ and have found $\mathrm{z}$ between 0.5 and 0.7 . The last limit corresponds to the case where dipolar forces dominate Brownian forces [3], [4]. A model of aggregation of Brownian magnetic particles in the linear chains has been developed in ref. [5]. Results of this model are in a good agreement with experiments. However the theoretical results were determined by the assumption of strong influence of the Brownian effects on the kinetics of the chain formation.

At the same time in many cases the Brownian phenomena in MRS are suppressed - the energy of magnetic interaction between the micron-sized particles is much larger than the thermal energy $k T$. In the presented work we consider kinetics of the chain formation in a system of magnetizable non-Brownian particles.

The structure of this paper is the following. In section 2 theoretical model of the aggregation process is presented. In section 3 direct computer simulations of the trajectories of the particles are carried out. Comparisons of the results of the computer simulations with that of analytical model are presented in the section 4.

\section{Theoretical model}

We consider suspension of identical non-Brownian magnetizable particles in a flat gap. Thickness of this gap is much less than the sizes of the boundary plates. The system is subjected to homogeneous magnetic field $\mathrm{H}$ perpendicular to the gap plane. We suppose that under the field action the particles aggregate into linear chains aligned along the field. This situation is illustrated in Fig.1.

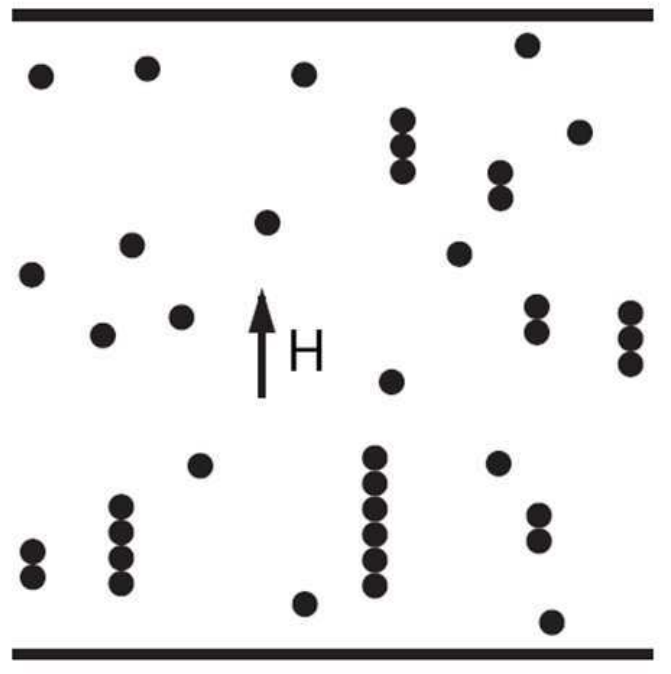

Fig.1

Sketch of the system under consideration

Let us denote by $\mathrm{g}_{\mathrm{n}}$ the number of $\mathrm{n}$-particle chains per unite volume of the system. Our aim is to determine the evolution of this function with time.

To this end we use the following approximations. First and foremost we neglect any Brownian effects in this system. It follows thence that we can also ignore destruction of the chains due to the thermal motion of the particles. This approximation is justified when the energy of magnetic interaction between particles is much more than the thermal energy $k T$. Second, we take into account only pair interaction between the chains, ignoring the simultaneous interaction between more than two chains. This approximation can be used when volume concentration $\varphi$ of the particles in the system is relatively low, about several per cent. It should be noted that for concentrated MRS one can expect the formation of dense solid-like bulk columns rather than that 
of the linear chains [1]. Theoretical analysis of the equilibrium phase transitions in MRS with formation of the dense phases of the particles is given in ref. [6]. The fact of appearance of only chains in the considered systems has been checked in our computer simulations.

Next, for the maximal simplification of the analysis, we suppose that the chains attach to each other only by their extremities and neglect their lateral aggregation.

In the framework of these approximations, the evolution of the population of chains can be represented by the following system of kinetic equations:

$$
\begin{aligned}
& \frac{d g_{n}}{d t}=\frac{1}{2} \sum_{k=1}^{n-1} \alpha_{n-k, k} g_{n-k} g_{k}-g_{n}\left[\sum_{l=1}^{N-n} \alpha_{n, l} g_{l}+\alpha_{n, n} g_{n} \Theta(N-2 n)\right] \\
& \Theta(x)=\begin{array}{l}
1, x \geq 0 \\
0, x<0
\end{array}
\end{aligned}
$$

Here $\alpha_{n m}$ is a kinetic coefficient of amalgamation of the $n$ - and $m$ - particle chains into a chain with $(n+m)$ particles, $N$ is the maximal possible number of particles in a chain. This number is determined by the gap thickness. Coefficient $1 / 2$ at the first term in eq.(1) is used to avoid counting twice the interaction between two chains of different sizes. The term with $k=n / 2$ (when $n$ is even) is taken into account two times in the first sum of (1), thus one time - in the first term of the right part of (1). The Heaviside function $\Theta$ as well as the upper limit of the sum in the square brackets in (1) implies that the maximum number of particles in a chain is $N$. The second term in these brackets takes into account that when two $n$-particle chains merge, two n-chains disappear.

According to their physical meaning, the coefficients $\alpha_{\mathrm{nm}}$ must be symmetrical with respect to the indexes $n$ and $m$. Then, one can show that equation (1) automatically satisfies to the normalization condition

$$
\sum_{n=1}^{N} n g_{n}=\text { const }
$$

Obviously the following equality const $=\varphi / \mathrm{V}$ must be fulfilled, where $\mathrm{V}$ is the particle volume, and $\varphi$ is the volume fraction of the particles.

Let us determine now the kinetic coefficient $\alpha_{k n}$. To this end we consider two chains consisting of $n$ and $k$ particles respectively (Fig.2).

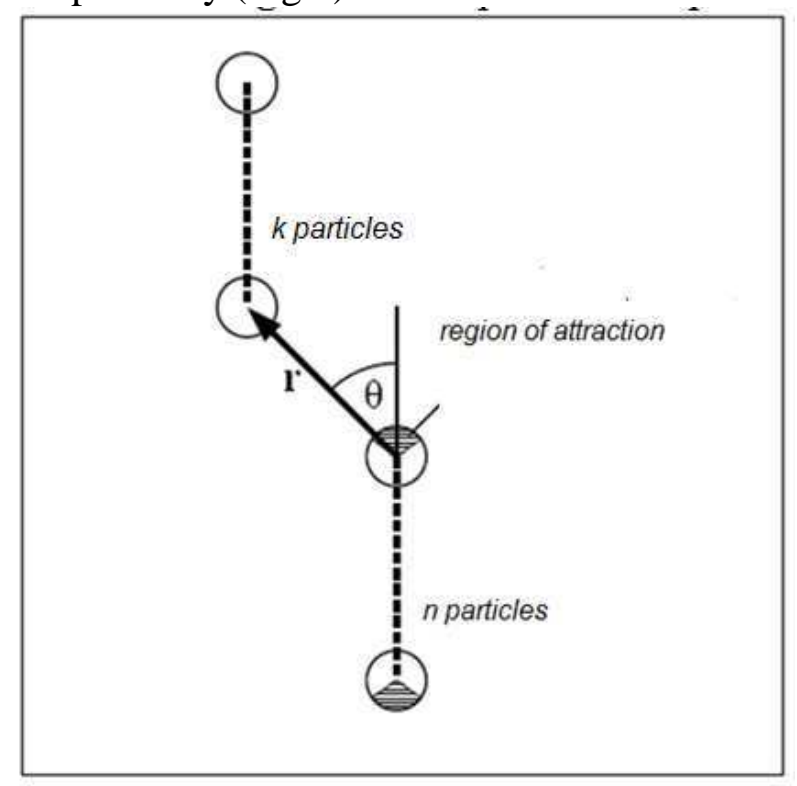

Fig.2. Sketch of the interacting chains 
We denote by $J_{k n}$ the flux of $k$-particle chains towards the $n$-particle one, i.e. $J_{k n} g_{n}$ is the number of $k$-particle chains which join to $n$-particle chains per unit time in a unit volume of the system.

In the framework of Eq.(1) we have:

$$
J_{k n}=\alpha_{k n} \cdot g_{k}
$$

(here there is no summation over index $k$ ).

On the other hand this flux is defined by

$$
J_{k n}=\int_{s_{\text {attr }}} G_{k}(\mathbf{r}) \cdot \mathrm{v}_{\mathrm{r}}(\mathbf{r}) d s
$$

Here $G_{k}$ is the number density of $k$-particle chains at the distance $\mathbf{r}$ from the $n$-particle chain, whereas $g_{\mathrm{k}}$ is the mean number of these chains in a unit volume; $\mathrm{v}_{\mathrm{r}}$ - is the relative radial velocity between a $k$-chain and a $n$-chain, $s_{\text {attr }}$ is the part of the surface of a particle at the extremity of the $n$-particle chain, corresponding to attraction between these chains. This situation is illustrated in Fig.2.

The main problem now lies in the fact that the function $\mathrm{G}_{\mathrm{k}}(\mathbf{r})$ is unknown. We need to express this function through the mean magnitude $g_{\mathrm{k}}$ which takes place in the kinetic equations (1).

An exact solution of this problem is very complicated and cumbersome. However the following physical considerations allow us to get simple estimates, which lead to reasonable agreement with the results of computer simulations.

Below we will discuss two kinds of approximations. The first one is simple and leads to good agreement with computer simulations for weakly concentrated systems with the particle concentration not more than 1-2\%. However, this model does not allow to describe kinetics of the aggregation of suspensions with the particle concentration of about 3-5\%.

The second approximation is a little more complicated, however leads to reasonable agreement with the simulations up to $5 \%$ of the particle concentration.

Weakly concentrated systems. The order of magnitude of the the mean distance $R$ between the nearest particles in suspension can be estimated as

$$
R \approx a \varphi^{-1 / 3}
$$

where $a=d / 2$ is the particle radius. It allows us to associate the mean concentration $g_{k}$ of the chains in suspension with the local concentration $G_{k}(R)$ at the distance $R$ from extremity of the $n$-particle chain. Strictly speaking, this distance between the $n$ - and $k$-particle chains must be determined by concentrations of these chains, not by the total particle concentration $\varphi$. However this modification would lead to more complicated calculations. We will see below that the simple estimate $R \approx a \varphi^{-1 / 3}$ leads to a good agreement with the computer simulations for weakly concentrated suspensions.

Due to the particle number conservation, the flux through the surface $s_{\text {attr }}$ is equal to the flux through the surface $S_{\text {attr }}$ at the average distance $R$. Thus, instead of (3) we can write down:

$$
J_{k n}=g_{k} \int_{S_{a t t r}} \mathrm{v}_{\mathrm{r}}(\mathbf{R}) d S
$$

Now we need to calculate the radial velocity $\mathrm{v}_{\mathrm{r}}$. This velocity can be determined from the equation 


$$
\mathrm{V}_{\mathrm{r}}(\mathbf{R})=-\left.\beta_{k n} \frac{\partial U_{k n}}{\partial r}\right|_{r=R}
$$

where $U_{\mathrm{kn}}$ is potential energy of interaction between these chains, $\beta_{\mathrm{kn}}$ is the Stokes coefficient of mutual mobility of the chains.

In order to estimate $U_{\mathrm{kn}}$ we will suppose that every particle in the chain has the same magnetic moment $m$ as a single particle in the magnetic field $\mathrm{H}$. In other words we will neglect the mutual interaction between the different dipoles of the chains. This approximation is widely used in the theory of magnetic suspensions [1]. In this approximation the energy $U_{\mathrm{kn}}$ equals to the sum of the dipole-dipole interaction of all $k$ particles of the first chain with all $n$ particles of the second one.

We can approximate this double sum by using the method of the image charges. When the distance between the chains is more than the diameter $d$ of the particle, the energy of the total dipole-dipole interaction between all particles in these chains formally is nearly equal to energy of the Coulomb interaction between four image charges $q= \pm m / d$ situated on the poles of the extremity particles of the chains [1]. These charges are shown in Fig. 3.

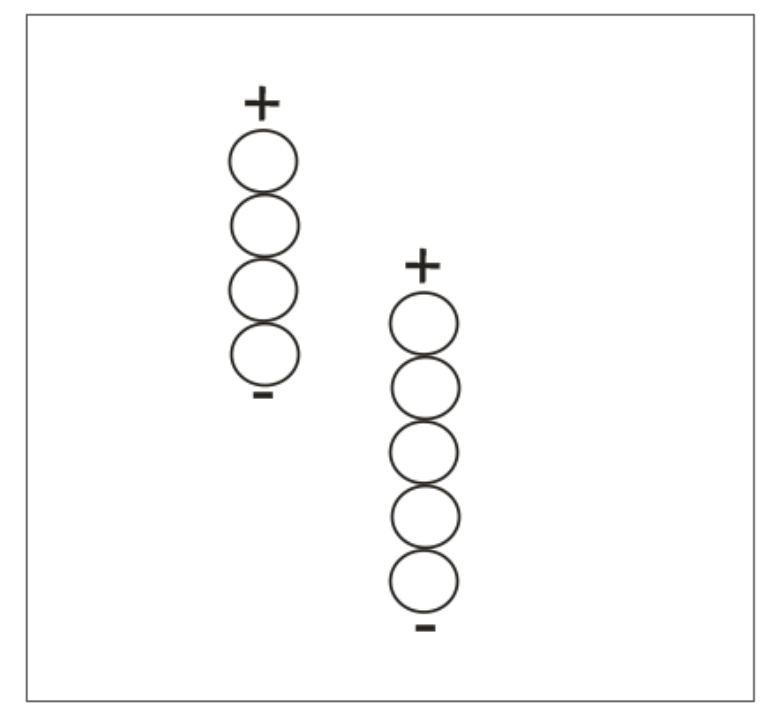

Fig.3. Image charges on the poles of the chains.

Because the total volume concentration $\varphi$ of the particles is supposed small, the distance $R_{\mathrm{kn}}$ between the particles on the chain extremities is much more than the particle diameter $d$. That is why the "four charge" approximation between the chains can be used instead of the double summation of the dipole-dipole interaction of all particles in the chains.

One can estimate the mobility coefficient $\beta_{k n}$ by modeling each $k$-particle chain as ellipsoid of revolution with the minor and major axes equal to $d$ and $k d$ respectively. The volume of this ellipsoid is equal to the total volume of the particles in the chain. Analytical expressions for the ellipsoid mobility coefficients are well known (see, for example, [7]). Generally speaking the ellipsoid mobility presents a tensor. For maximal simplification of calculations, taking into account that at the moment of aggregation, the axes of the chains are nearly parallel to the line of attraction force between them, we will use component of this tensor corresponding to the motion along the ellipsoid major axis. Keeping in mind that $\beta_{k n}$ corresponds to the relative velocity of two attracting chains, we get [7]: 


$$
\begin{array}{ll}
\beta_{k n}=\left(\beta_{\mathrm{k}}+\beta_{\mathrm{n}}\right) & \beta_{l}=\frac{1}{16 \pi \eta}\left(\chi_{l}+d^{2} n^{2} \gamma_{l}\right) \\
\chi_{l}=\int_{0}^{\infty} \frac{d \lambda}{\Delta(\lambda)} & \gamma_{l}=\int_{0}^{\infty} \frac{d \lambda}{(l d+\lambda) \Delta_{l}(\lambda)} \quad \Delta_{l}(\lambda)=\sqrt{\left(l^{2} d^{2}+\lambda\right)\left(d^{2}+\lambda\right)\left(d^{2}+\lambda\right)}
\end{array}
$$

Having estimated $U_{k n}$ in the approximation of four image charges and using Eq. (6), we come to the following expression for the radial relative velocity of the chains

$$
\begin{aligned}
& \mathrm{v}_{\mathrm{r}}=\frac{\beta_{k n}}{4 \pi \mu_{0}}\left(\frac{m}{d}\right)^{2}\left[-\frac{r-d \operatorname{Cos} \theta}{\left(r^{2} \operatorname{Sin}^{2} \theta+(r \operatorname{Cos} \theta-d)^{2}\right)^{\frac{3}{2}}}-\frac{r+d(k+n-1) \operatorname{Cos} \theta}{\left(r^{2} \operatorname{Sin}^{2} \theta+(r \operatorname{Cos} \theta+d(k+n-1))^{2}\right)^{\frac{3}{2}}}+\right. \\
& \left.+\frac{r-d(k-1) \operatorname{Cos} \theta}{\left(r^{2} \operatorname{Sin}^{2} \theta+(r \operatorname{Cos} \theta+d(k-1))^{2}\right)^{\frac{3}{2}}}+\frac{r-d(n-1) \operatorname{Cos} \theta}{\left(r^{2} \operatorname{Sin}^{2} \theta+(r \operatorname{Cos} \theta+d(n-1))^{2}\right)^{\frac{3}{2}}}\right]
\end{aligned}
$$

The angle $\theta$ is illustrated in Fig.1.

It is convenient to define the formal velocity of the chain attraction as:

$$
\mathrm{v}_{r}^{*}=\left\{\begin{array}{c}
\mathrm{v}_{\mathrm{r}}, \mathrm{v}_{\mathrm{r}} \leq 0 \\
0, \mathrm{v}_{\mathrm{r}}>0
\end{array} .\right.
$$

It has been noted that we neglect lateral aggregation of chains. Therefore we consider only the positions of the chains corresponding to $\theta<\pi / 2$.

The flux $J_{k n}$ at a distance $r=R$ is:

$$
J_{k n}=g_{k} \int_{S_{a t t r}^{*}} \mathrm{v}_{r}^{*} d S
$$

Combining relations (2), (6) and (8), we come to the following estimate:

$$
\alpha_{k n}=\int_{S_{\text {atr }}^{*}} \mathrm{v}_{r}^{*} d S
$$

The integrals in (10) can be easily calculated numerically.

Moderately concentrated suspensions. Our analysis shows that the estimate of the distance between interacting chains $R=a \varphi^{1 / 3}$ leads to a bad agreement with results of computer simulations when concentration of particles exceeds three percent. The reason for this disagreement lies in the fact that the mean distance between chains with $n$ and $k$ particles depends on concentrations $g_{n}$ and $g_{k}$ of these chains. This is not significant when the particle volume concentration is about one percent, however it becomes important for higher concentrations. To take it into account we will estimate the characteristic distance between the chains as

$$
R_{k n} \sim\left(\max \left(g_{k} ; g_{n}\right)\right)^{-1 / 3}
$$


The numerical multiplier in the right part of (11) cannot be determined from simple considerations but can be found from comparison of the analytical calculations with the results of laboratory or computer experiments.

After this modification of the characteristic distance between chains, the formulas (8-10) for the radial velocity $\mathrm{v}_{\mathrm{r}}$, flux $J_{k n}$ and the kinetic coefficient $\alpha_{k n}$ formally do not change. However now the surface of integration $S_{a t t r}$ corresponds to the part of spherical surface with the radius $R_{k n}$ on which the radial velocity $\mathrm{v}_{\mathrm{r}}$ is negative. Therefore, the position of this surface as well as the kinetic coefficients $\alpha_{k n}$ depend on the concentrations $g_{n}$ and $g_{k}$.

Now we need to estimate the particle magnetic moment $m$. Under assumption of constant permeability $\mu_{\mathrm{p}}$ of the particle, we have [8]:

$$
m=\frac{\pi \mu_{0} d^{3}}{2} \frac{\mu_{p}-1}{\mu_{p}+2} H
$$

where $\mu_{0}$ is the vacuum permeability. Substituting (12) into (8-10), and, then into (1), we come to the final form for the equations of evolution of the size distribution. Assuming that at the onset of aggregation all particles are isolated, we get the following initial conditions for eqs. (1):

$$
t=0, \quad g_{n}=\frac{1}{V} \varphi \delta_{n 1}
$$

Here $\delta_{n 1}$ is the Kroneker symbol.

The equations (1), (13) can be solved numerically.

\section{Computer simulations.}

Three-dimensional computer model of the MRS aggregation requires too long time of calculations. In order to verify the main ideas of the analytical model, we use here a twodimensional computer model. In the framework of this model the disk-shaped particles are situated in a plane parallel to the applied magnetic field $\mathbf{H}$. Magnetic moment of the particle in the computer simulation, like in the analytical model, is calculated by the formula (12). It should be stressed that we use the two-dimensional simulation only to verify the analytical model, not to describe a real system of magnetic disks. Therefore we can use here the formula (12), which is valid for magnetizable spheres.

In the simulation we take into account magnetic interactions between all particles and ignore hydrodynamical interactions between the particles.

Neglecting inertia, the equation of motion of the $i$-th particle is:

$$
\frac{d \mathbf{r}_{i}}{d t}=\frac{1}{3 \pi \eta d}\left(\sum_{j \neq i}\left(\mathbf{F}_{m}^{i, j}+\mathbf{F}_{s t}^{i, j}\right)\right.
$$

Here $\mathbf{r}_{i}$ is the position of $i$-th particle, $\mathbf{F}_{m}^{i, j}$ and $\mathbf{F}_{s t}^{i, j}$ are the magnetic and sterical forces of interaction between the $i$-th and $j$-th particles.

In the framework of the dipole-dipole interaction, the radial and tangential components of the magnetic force can be calculated as:

$$
\begin{aligned}
F_{m, r}^{i, j} & =\frac{3 m_{i} m_{j}}{4 \pi \mu_{0}} \frac{1-3 \cos ^{2} \theta}{r_{i, j}^{4}} \\
F_{m, \theta}^{i, j} & =-\frac{3 m_{i} m_{j}}{4 \pi \mu_{0}} \frac{2 \sin \theta \cos \theta}{r_{i, j}^{4}}
\end{aligned}
$$


Here $r_{i, j}$ is the distance between the particle centers, $m_{i}$ and $m_{j}$ are their magnetic moments, $\theta$ is the angle between the applied field $\mathbf{H}$ and the radius vector $\mathbf{r}_{\mathrm{ij}}$.

The short range force of sterical interaction between particles preventing their interpenetration was simulated as follows. In the program of the computer experiment, upon the interpenetration of particles $\left(r_{i j} \leq d\right)$, the repulsive force was applied to particles, the value of which makes them to leave the region of interpenetration during one time step. At the same time, the force of magnetic attraction was equated to zero under condition $r_{i j} \leq d$. As soon as the particles separate under the repulsion force $\left(r_{i j}>d\right)$, we put this force equal to zero and reintroduce the magnetic force.

We have used about 4000 particles in the computer simulations and solved numerically equations (14) for all particles. At the onset of simulations a random particle distribution on the plane has been created. The time step has been chosen so that the particle displacement for a given step would not exceed $d / 20$. The particles are in a square region which is determined by repulsive boundary conditions. Some results of our simulations are illustrated in Figs. 4 and 5. The viscosity of the carrier liquid, size of the particles and the magnetic field correspond to experiments made with nickel particles that we are currently doing in order to test this model.

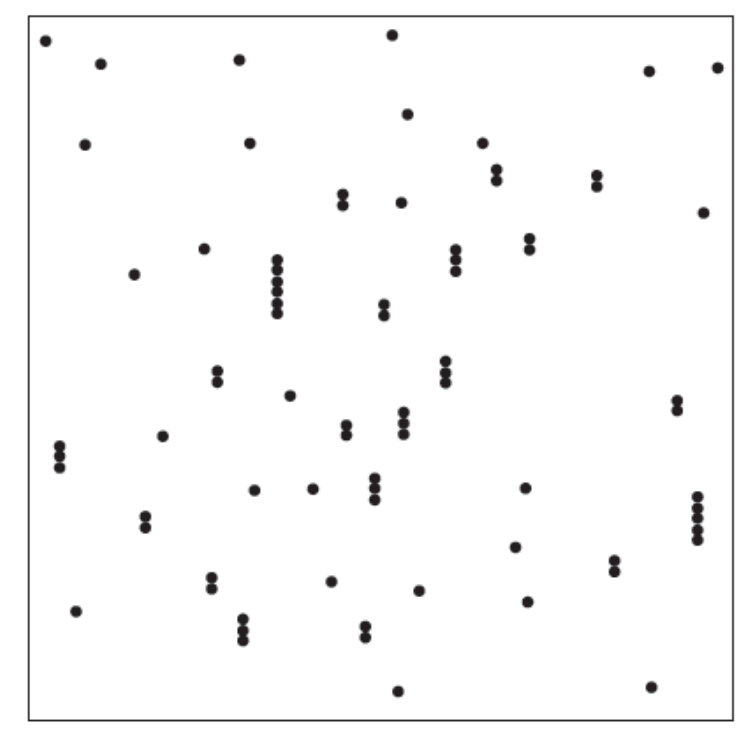

Fig.4

The snapshots of the simulations for the time $t_{1}=450 \mathrm{~s}$ after beginning of aggregation. Parameters of the system: magnetic field $\mathrm{H}=13130 \mathrm{~A} / \mathrm{m}$; the carrier liquid viscosity $\eta=20 \mathrm{~Pa} \cdot \mathrm{s}$; diameter of the nickel particle $3 \mu \mathrm{m}$; the surface concentration of the disks $f=0.014$. 


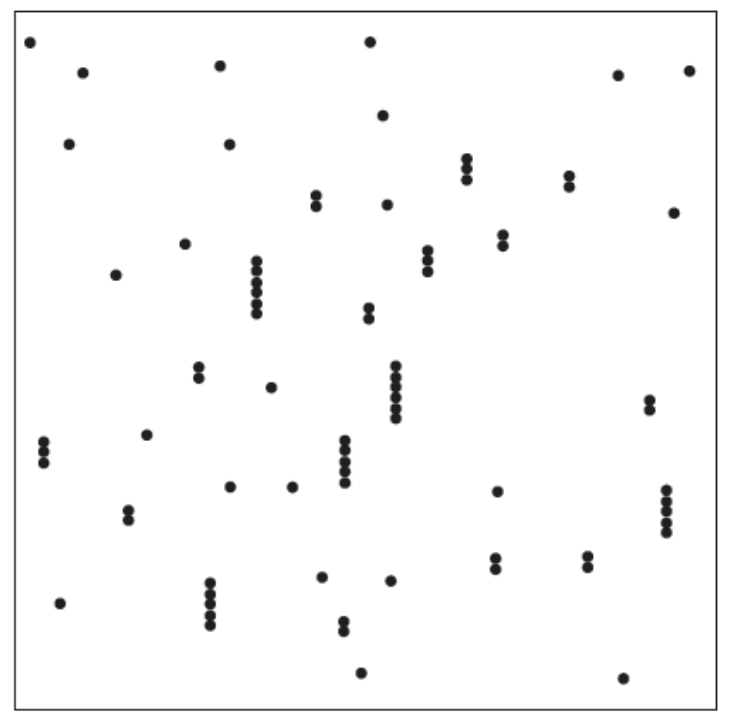

Fig.5

Same as in Fig. 4 for the time $t_{2}=900 \mathrm{~s}$ after beginning of aggregation

\section{Comparison between theory and computer simulations}

In this part we compare results of the analytical model with the simulations.

It was noted in the part 3 that in order to avoid too long time of calculations, the simulations have been carried out in two-dimensional approximation, in which the particles were modeled as disks able to move only in the plane, parallel to the applied field.

In order to adapt $3 \mathrm{D}$ theoretical model to $2 \mathrm{D}$ computer simulations, we need to make the following transformations. First, instead of the volume concentration $\varphi$ of the spherical particles we must use the surface concentration $f$ of the disks. Second, in the approximation of weakly concentrated suspension the mean distance $R$ between chains instead of (4) can be estimated as

$$
R \approx a f^{-1 / 2}
$$

Third, while calculating the flux $J_{k n}$ in the expression similar to (9), one should integrate $\mathrm{v}_{r}^{*}$ over an arc $l_{\text {atr }}^{*}$, on which the chains attract $\left(\mathrm{v}_{\mathrm{r}}<0\right)$. Next, instead of volume of the spherical particle $\mathrm{V}$, the cross section $\mathrm{S}$ of the particle must be used.

As a result, the kinetic equations for the 2D model have the same form (1) as for the 3D case, however the coefficient $\alpha_{k n}$ should be calculated by the formula

$$
\alpha_{k n}=\int_{l_{\text {attr }}^{*}} \mathrm{v}_{r}^{*} d l
$$

instead of eq.(10). Integral in (17) can be calculated numerically. As in the 3D case, we integrate over the region $-\pi / 2<\theta<\pi / 2$.

Some results of the computer simulations as well as numerical solutions of the model $(1,16,17)$ for the weakly concentrated suspension are presented in Fig. 6 . The expressions for coefficient $\beta_{k n}$ of the mutual mobility of the chains, for the modified radial velocity $\mathrm{v}_{r}^{*}$ as well as for the particle magnetic moment $m$ have been used in the forms (7), (8) and (12) in both cases of the analytical model and computer simulations. 


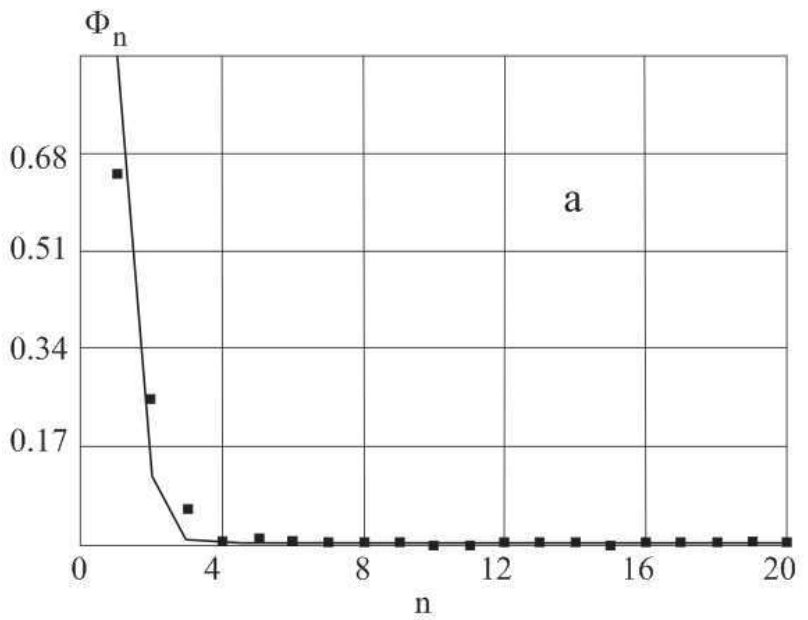

n

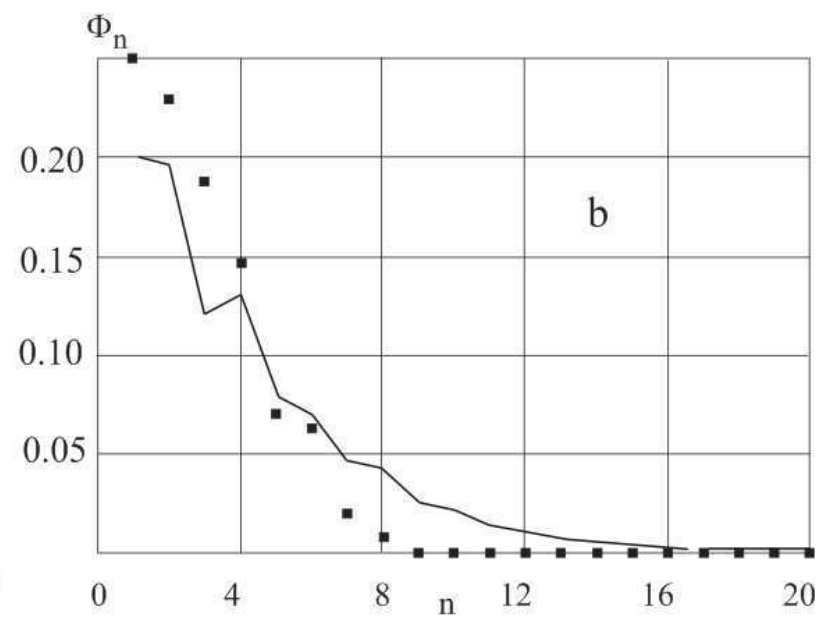

Fig.6

Relative surface concentration $\Phi_{n}=g_{n}\left(t_{1}\right) n S / f$ of the $n$-particle chain vs. number $n$ of particles in the chain, $S$ is the cross section of the particle area, $f$ is the total surface concentration of particles. Physical parameters of the system are the same as in Fig. 4 and Fig. 5. Solid lines - results of analytic model, dots - simulations. $f=0.014$. a) Time $t$ after the field was switched on, is $50 \mathrm{~s}$; b) $t=900 \mathrm{~s}$. Size of dots equals to the simulation error bar.

Theoretical and computer results are in quite good agreement for any time after onset of the aggregation. The zigzags of the solid lines (analytical model) here and below appear because we deal with integer variations of $n$.

Modification of the model to the moderately concentrated systems leads to the estimate $R_{k n}=C \cdot\left(\max \left(g_{k} ; g_{n}\right)\right)^{-1 / 2}$ instead of eq.(11). The numerical multiplier $C$ has been fitted by comparison of analytical calculations and computer simulations. As a result the form has been chosen:

$$
R_{k n}=\frac{1}{2}\left(\max \left(g_{k} ; g_{n}\right)\right)^{-1 / 2}
$$

Figures 7, 8 show distribution functions $g_{n}$ calculated by the model (1), (17), (18) and results of computer simulations with the surface concentrations $f=0.014$ and $f=0.05$ respectively. 

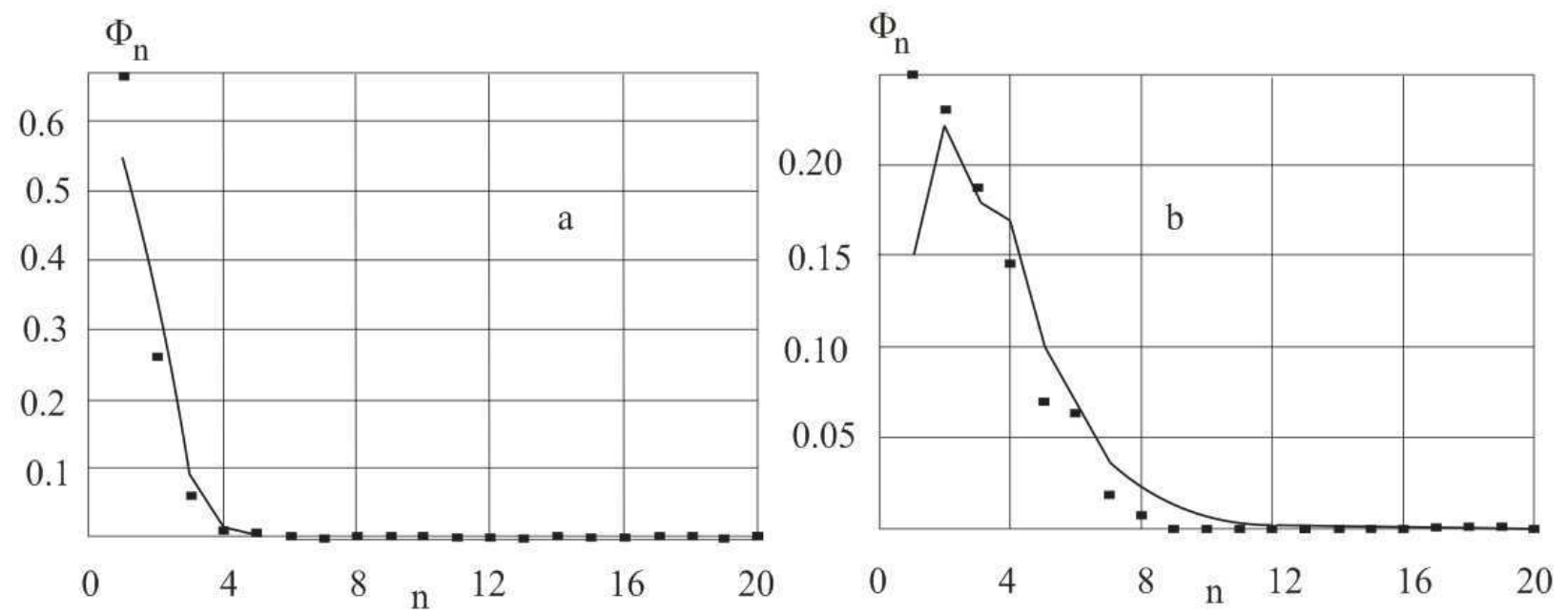

Fig.7

Same as in Fig.6a; analytical calculations (solid line) made in the approximation (18) of moderately concentrated system instead of (16); $f=0.014$; a) $t=50 \mathrm{~s}$; b) $t=900 \mathrm{~s}$

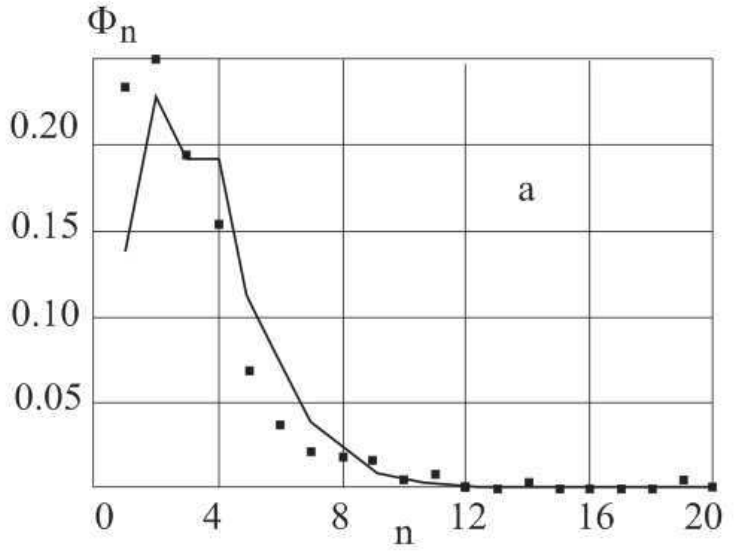

Fig.8

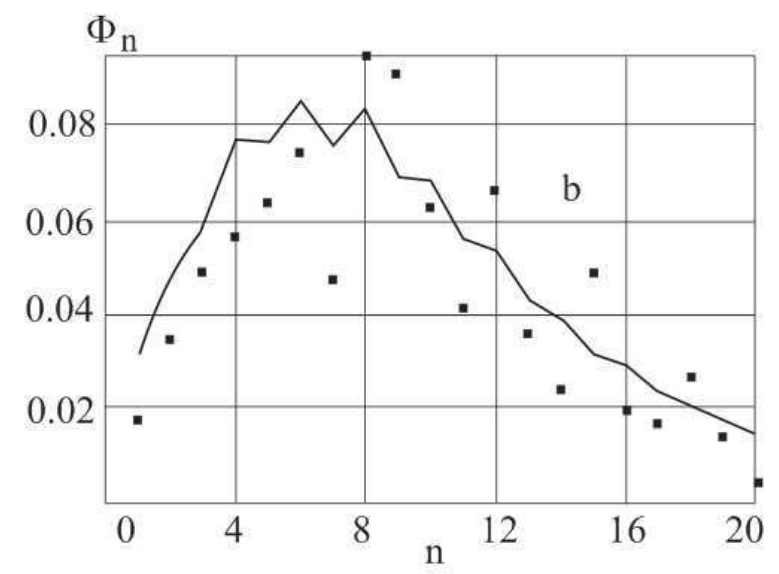

Same as in Fig.7 for the area fraction $f=0.05$. a) $t=50 \mathrm{~s}$; b) $t=900 \mathrm{~s}$

In spite of the strong simplifications, the theoretical model leads to reasonable agreement with the computer simulations. For the systems with the concentration $f$ of about 0.014 both models (16) and (18) are quite accurate, however the model (16) is significantly simpler for calculations. That is why it can be recommended for analysis of chaining in weakly concentrated systems.

\section{Conclusion}

Theoretical model of kinetics of evolution of chain-like aggregates in suspensions of nonBrownian magnetizable particles is proposed. In spite of strong simplifications, results of this model are in reasonable agreement with results of computer simulations for the two-dimensional 
version of the model, when the surface concentration of the particles is about several per cent. This model can be considered as a robust basis for theoretical modeling of kinetics of the chaining in low- and moderately concentrated magnetorheological and other polar suspensions of nonBrownian particles. In particular it can be used to study conductivity percolation in composites in which metal particles form chains aligned along magnetic field. Since the branch-like, dense dropand solid-like aggregates are ignored in this model, it cannot be applied to concentrated suspensions where these bulk structures are quite typical. A further step in the model would be to take into account lateral agregation between chains which should increase its range of applicability. Such improvement is under consideration as well as experiments with a monolayer of magnetizable particles.

\section{Acknowledgements}

This work has been supported grants of Russian Agency of Education 2.1.1/2571 and 2.1.1/1535, grants of Russian Federal Target Program 02.740.11.0202, № 02-740-11-5172 and NK-43P(4); grants of Russian Fund of Fundamental Investigations 10-01-96002-Ural, 10-0296001, 10-02-00034 


\section{References}

1. G. Bossis, O. Volkova., S. Lacis., A. Meunier. In Ferrofluids Magnetically Controlable Fluids and Their Applications, Ed. S.Odenbach. Springer 2002.

2. H.See, M.Doi, J.Phys.Soc.Japan, (1991),60 ,2778-2782

3. E.Climent, M.R.Maxey, G.E.Karniadakis, Langmuir,2004,20,507-513

4. M.C.Miguel, R.P.Satorras, Phys.Rev.E,1999,59,826

5. G. Bossis, C. Métayer, A. Zubarev, Phys.Rev.E, 2007, V. 76. P. 041401

6. L.Yu. Iskakova, A.P. Romanchuk, A.Yu. Zubarev, Physica A., 2006 V. 366. P.18

7. J. Happel, H.Brenner, Low Reynolds Number Hydrodynamics, Prentice-Hall, Englewood Cliffs, NJ,1965

8. L.D.Landau, E.M.Lifshitz, Electrodynamics of Continuum Media, Pergamon Press, London, 1960

\section{Figure captures}

Fig.1. Sketch of the system under consideration

Fig.2. Sketch of the interacting chains

Fig.3. Image charges on the poles of the chains.

Fig.4. The snapshots of the simulations for the time $t_{1}=450 \mathrm{~s}$ after beginning of aggregation.

Parameters of the system: magnetic field $\mathrm{H}=13130 \mathrm{~A} / \mathrm{m}$; the carrier liquid viscosity $\eta=20 \mathrm{~Pa} \cdot \mathrm{s}$; diameter of the nickel particle $3 \mu \mathrm{m}$; the surface concentration of the disks $\varphi=0.014$.

Fig.5. Same as in Fig 4, for the time $\mathrm{t}_{2}=900 \mathrm{~s}$ after beginning of aggregation

Fig.6. Relative surface concentration $\Phi_{n}=g_{n}\left(t_{1}\right) n S / f$ of the $n$-particle chain vs. number $n$ of particles in the chain. $S$ is the cross section of particle area, $f$ - the total surface concentration of particles.

Physical parameters of the system are the same as in Fig. 4 and Fig. 5. Solid lines - results of analytic model, dots - simulations. $f=0.014$. a) Time $t$ after the field was switched on, is $50 \mathrm{~s}$; b) $t=900 \mathrm{~s}$. Size of dots equals to the simulation error bar.

Fig.7 Same as in Fig.6a; analytical calculations (solid line) made in the approximation (18) of moderately concentrated system instead of (16); $f=0.014$; a) $t=50 \mathrm{~s} ; \mathrm{b}) t=900 \mathrm{~s}$

Fig.8. Same as in Fig.7 for the area fraction $f=0.05$. a) $t=50 \mathrm{~s}$; b) $t=900 \mathrm{~s}$ 
